\title{
Case report \\ Endoscopic foley catheter technique for removal of esophageal foreign body
}

\begin{abstract}
Impacted esophageal foreign body in children may pose a challenging clinical scenario to the gastroenterologists. Early recognition and treatment is imperative as the complications can be life threatening. The Foley catheter method of foreign body extraction has been used on some patients, but endoscopy remains the treatment modality of choice. Here we report an interesting case of a child with impacted stone in the esophagus in whom a combined endoscopic-Foley catheter technique was successfully applied to extract the impacted foreign body
\end{abstract}

Keywords: endoscopy, esophageal, foley catheter, foreign body
Volume 6 Issue 2 - 2017

\author{
Saket Kumar, Amit Dangi, Abhijit Chandra \\ Department of Surgical Gastroenterology, King George's \\ Medical University, India
}

Correspondence: Abhijit Chandra, Department of Surgical Gastroenterology, King George's Medical University, Lucknow, Uttar Pradesh, India, Tel 915222256116, Fax +91-522-2256116, Email mailto:bhijitchandra@hotmail.com

Received: December 28, 2016 | Published: February 21, 2017
Abbreviations: FB, foreign body; FFCT, fluoroscopic foley catheter technique

\section{Introduction}

Esophageal Foreign body (FB) impaction is common in paediatric age group. Endoscopic removal of the FB is considered the standard treatment, while non-endoscopic methods have also been described in literature. ${ }^{1}$ Non-endoscopic methods such as a Foley catheter technique with or without fluoroscopy have certain advantages in terms of simplicity and low-cost of the procedure, particularly useful for proximally impacted FBs. However, it has not been uniformly accepted in clinical practice, as it does not allow direct visualisation of the esophagus and has lower success rate.

We are reporting a case of endoscopic Foley catheter technique for removal of an esophageal foreign body done under direct vision when classical endoscopic techniques like forceps removal failed. This innovative technique can be used to remove esophageal foreign body that are not sharp and faceted.

\section{Case presentation}

A 7-year old girl was referred to us with complain of absolute dysphagia. She had history of accidental pebble ingestion 13 days back. At the time of presentation, the child was conscious, dehydrated but had no respiratory distress. She was unable to swallow her saliva and she was complaining of retrosternal chest pain. A general physical examination was normal. A plain radiograph of the chest revealed a rounded radio-opaque object measuring around $2.5 \times 3.5 \mathrm{~cm}$ located at the level of mid esophagus (Figure 1).

An esophagoscopy done under general anaesthesia revealed a white, round marble stone impacted at the mid-oesophagus. Forceps/ snare removal was not successful because of the round shape and smooth surface of the stone. A 14 Fr Foley catheter was guided post foreign body and balloon was inflated with $5 \mathrm{ml}$ saline. The Foley catheter was gradually pulled out along with the stone under endoscopic vision. The intermittent suction applied endoscopically also helped dislodgement and proximal movement of the stone. The stone was then brought to the oral cavity from where it was finally removed. Check endoscopy showed intact esophageal mucosa. The patient was given oral diet after recovering from anaesthesia, which she tolerated well. She was discharged from the hospital next day.

\section{Discussion}

FB ingestion is a frequent complaint in paediatric emergency services. Most of the ingested FBs pass out of the gastrointestinal tract without getting impacted and intervention (endoscopic or surgical) is required only in $10 \%-20 \%$ of the cases. ${ }^{2}$ The esophagus is the narrowest area of the digestive tract, which also makes it the commonest site of FB impaction. An esophageal foreign body may lodge in the thoracic inlet, the aortic arch area, or the GE junction. In our case also the FB was lodged in the mid-esophagus, coinciding with aortic arch indentation. Commonly ingested objects among children include coins, button batteries, toys, magnets, pins, marbles or pebbles and food boluses etc. ${ }^{1-3}$ [Our patient presented with history of accidental ingestion of pebble, which is commonly ingested FB in this part of the world.

Choking and dysphasia are the most common presentations in cases of esophageal FB impaction [1]. Other symptoms include odynophagia, diffuse chest pain, respiratory difficulties. Our patient had absolute dysphasia without any cough, fever or respiratory distress. This presentation may be attributed to round shape of the stone, which completely blocked the esophageal lumen without causing significant mucosal damage because of its smooth surface.

Whenever a child presents with foreign body ingestion, a simple $\mathrm{X}$-ray neck, chest and abdomen is recommended. ${ }^{3}$ It readily detects the radiopaque foreign bodies and sometimes may also diagnose associated complication like esophageal perforation. Radiographic contrast is usually avoided due to risk of aspiration. In few patients, in whom there is suspicion of esophageal perforation, computed tomography proves very useful in deciding the management plan [3]. In our case also, chest X-rays confirmed the presence and the location of the ingested FB (Figure 1).

Endoscopic removal of the impacted FB is the standard treatment option, but some small, blunt objects may also be extracted using a Foley catheter. Flexible endoscopy is the "first line" approach for upper gastrointestinal foreign bodies with a success rate of between $80 \%-100 \%$ and a less than $1 \%$ risk of perforation. ${ }^{4}$ Various endoscopic retrieval devices are available that can be used to remove the foreign body (polypectomy snares, rat-tooth and alligator forceps, Dormia baskets, magnetic probes polyp graspers, retrieval nets, and frictionfit adaptors or banding caps). Surgical intervention is indicated only when endoscopic retrieval fails or is contraindicated. ${ }^{5,6}$ In the present 
case, due to the smooth and round form of the FB, endoscopy with both forceps and Dormia basket failed to retrieve the stone.
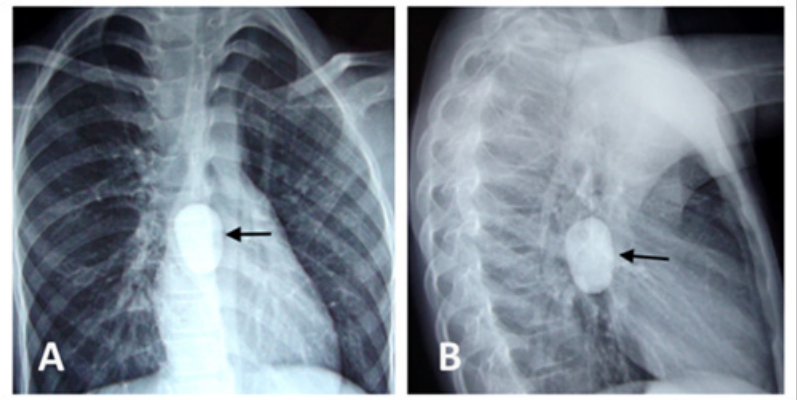

Figure I Chest X-Ray posterior-anterior view demonstrating radio-dense foreign body at the level of mid esophagus (A), Chest X-ray lateral view of the same patient $(B)$.

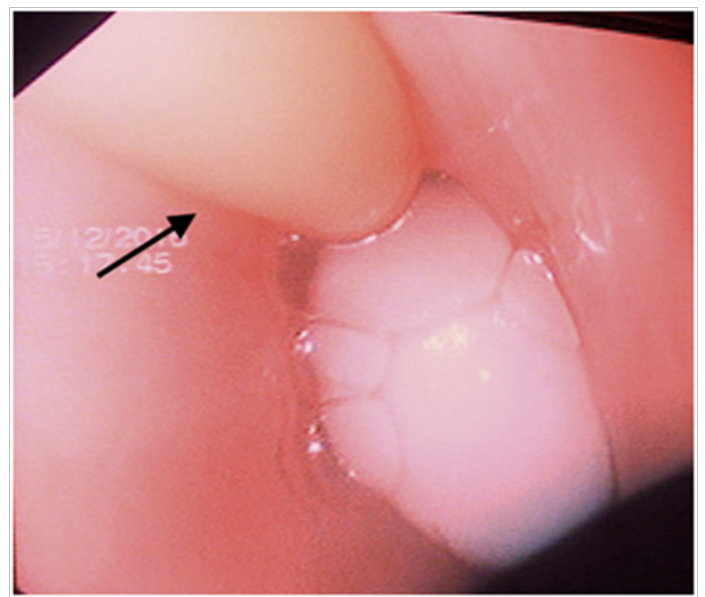

Figure 2 Endoscopic picture of Foley catheter (arrow) retrieving the foreign body.

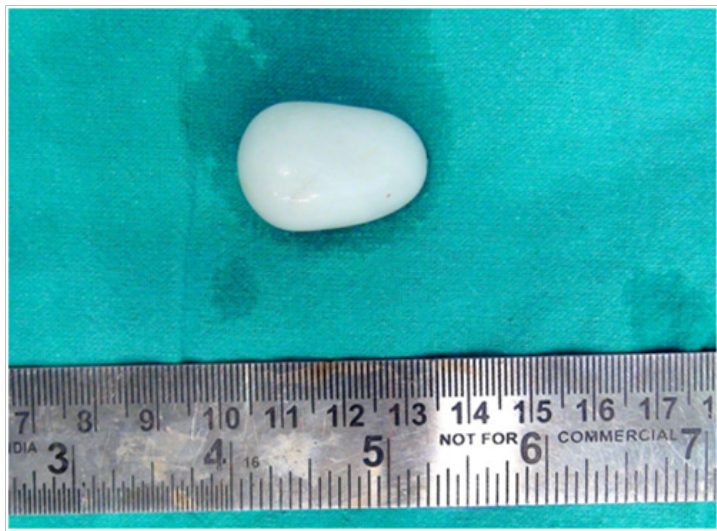

Figure 3 Retrieved pebble of size approximately $3 \times 2 \mathrm{~cm}$.

Several non-endoscopic methods of removing foreign bodies have been successfully used in the past. These include esophageal bougienage, the penny-pincher technique and Foley catheter technique (fluoroscopic guided or blind). Esophageal bougienage involves pushing the foreign body into stomach by passing an esophageal dilator. It has been used successfully at different centres with high reported success rate (94\%-95\%).,7 The penny-pincher technique involves fluoroscopy-guided insertion of a grasping endoscopic forceps though a soft rubber catheter into the esophagus. No sedation or placement of an advanced airway device is required in these techniques. ${ }^{4,7,8}$

Schunk et al. have reported the safety and efficacy of the fluoroscopic Foley catheter technique (FFCT) for esophageal FB in children. In their study, the FFCT was successful in $91 \%$ (378 of $415)$ of the cases with very few procedure-related complications. ${ }^{9}$ Similar studies have shown that FFCT is easy to perform, requires no anaesthesia, reduces hospital stay and cost of treatment. ${ }^{9,10}$ However, there are certain contraindications of this technique where alternative modality is advised. These include- impaction of more than 72 hours, three unsuccessful removal attempts; complete obstruction of the esophagus; esophageal perforation; signs of respiratory distress; children younger than 1.5 years; sharp-edged foreign bodies; and button batteries that have been impacted for more than 2 hours. ${ }^{11}$

Despite having several advantages, non-endoscopic techniques are not widely accepted in the western practice as they do not allow direct visualization the esophagus and there is theoretical risk of severe complications such as broncho-aspiration, esophageal perforation, and acute airway obstruction. For these reasons, it is recommended that removal of foreign bodies should be carried out under direct vision with airway protection, especially in the paediatric age group.1 In our case, we used a 14 Fr Foley catheter and stone was retrieved under direct vision of a flexible endoscope while protecting the airway, therefore completely eliminating the blind nature of fluoroscopic Foley catheter technique. Also, since Foley catheter was used in place of an expensive endoscopic esophageal balloon, the cost of the procedure was significantly decreased.

Universal availability, low-cost, and a relatively risk-free procedure make endoscopic Foley catheter technique an attractive option for retrieval of blunt, non-impacted esophageal FBs. This assumes more relevance in those parts of world where advance endoscopic facility is confined to tertiary health care centres and is out of reach of poor patients. It can also be successfully used in cases when conventional endoscopic manoeuvres like forceps/snare removal fails as in our case.

\section{Conclusion}

Endoscopic Foley catheter technique has the combined advantage of both endoscopic and Foley catheter techniques. It allows retrieval of FB under vision and in a simple, inexpensive way. This technique may also prove useful in emergency situation where specialized endoscopic balloon is not readily available.

\section{Funding}

None.

\section{Acknowledgments}

None.

\section{Conflicts of interest}

The authors declare no conflict of interest

\section{References}

1. Little DC, Shah SR, St Peter SD, et al. Esophageal foreign bodies in the pediatric population: our first 500 cases. J Pediatr Surg. 2006;41(5):914 918. 
2. Burgos A, Rabago L, Triana P. Western view of the management of gastro esophageal foreign bodies. World $J$ Gastrointest Endosc. 2016;8(9):378-384.

3. Ikenberry SO, Jue TL, Anderson MA, et al. Management of ingested foreign bodies and food impactions. Gastrointest Endosc. 2011;73(6):1085-1091.

4. Heinzerling NP, Christensen MA, Swedler R, et al. Safe and effective management of esophageal coins in children with bougienage. Surgery. 2015;158(4):1065-1070.

5. Louie MC, Bradin S. Foreign body ingestion and aspiration. Pediatr Rev. 2009;30(8):295-301.

6. Uyemura MC. Foreign body ingestion in children. Am Fam Physician 2005;72(2):287-291.

7. Allie EH, Blackshaw AM, Losek JD, et al.Clinical effectiveness of bougienage for esophageal coins in a pediatric ED. Am J Emerg Med. 2014;32(10):1263-1269.
8. Gauderer MW, DeCou JM, Abrams RS, et al. The 'penny pincher': a new technique for fast and safe removal of esophageal coins. $J$ Pediatr Surg. 2000;35(2):276-278.

9. Schunk JE, Harrison AM, Corneli HM, et al. Fluoroscopic Foley Catheter Removal of Esophageal Foreign Bodies in Children: Experience With 415 Episodes. Pediatrics. 1994;94(5):709-714.

10. Morrow SE, Bickler SW, Kennedy AP, et al. Balloon extraction of esophageal foreign bodies in children. J Pediatr Surg. 1998;33(2):266270

11. Gasior AC, Knott EM, Sharp SW, et al. Predictive factors for successful balloon catheter extraction of esophageal foreign bodies. Pediatr Surg Int. 2013;29(8):791-794. 